\title{
Scanning Electron Microscopy Preparation Method for Coolia sp., a Delicate Dinoflagellate
}

\author{
Gómez-Lizárraga Laura Elena ${ }^{1, *}$, Durán-Riveroll Lorena María ${ }^{2}$ and Pérez-López Hugo Isidro ${ }^{3}$ \\ 1. Servicio Académico de Microscopía Electrónica de Barrido, Instituto de Ciencias del Mary \\ Limnología, Universidad Nacional Autónoma de México, Ciudad de México, México. \\ 2. Catedrática CONACYT, Instituto de Ciencias del Mar y Limnología, Universidad Nacional Autónoma \\ de México, Ciudad de México, México. \\ 3. Unidad Académica Procesos Oceánicos y Costeros, Instituto de Ciencias del Mar y Limnología, \\ Universidad Nacional Autónoma de México, Ciudad de México, México. \\ * Corresponding author: lauragomliz@cmarl.unam.mx
}

One of the recurrent problems in scanning electron microscopy (SEM) processing techniques is the distortion of biological material. This problem occurs mostly during sample drying step [1]. In this study, we present a simple and effective SEM protocol for preparation and observation of Coolia sp. a delicate benthic dinoflagellate. Delicate dinoflagellates species lack thick cellulosic plates, which makes them highly susceptible to distortion such as shrinkage and collapsing of cell structures during standard SEM preparation protocols $[2,3]$.

Biological samples standard methodology included: Fixation (preservation of cellular structure with no alteration from living state and halt potentially destructive autolytic processes). Dehydration (gradually replacement of water within the cell by graded series of organic solvent such as ethanol or acetone). Drying (by critical-point drying CPD method or chemical drying agents like hexamethyldisilaze HMDS). Coating with a thin electro-conductive material and SEM examination $[1,4]$.

Preservation of morphological features of delicate dinoflagellates without distortion of the cells is possible by applying different protocols $[2,3]$. We proposed the following preparation technique: Monospecific cells of dinoflagellates cultured in modified GSe medium, prepared with sterile seawater, were fixed with $2 \%$ glutaraldehyde in $1.5 \mathrm{~mL}$ of liquid medium for 90 minutes. Samples were washed and centrifuged (8000 rpm, $5^{\circ} \mathrm{C}$, for 6 minutes) four times in $1.5 \mathrm{~mL}$ of cold distilled water $\left(5^{\circ} \mathrm{C}\right)$ to prevent formation of $\mathrm{NaCl}$ crystals from seawater. Samples underwent a graded dehydration series of ethanol (10, 30, 50, 70, 90 and $99 \%, 1.5 \mathrm{~mL}$ each) and centrifuged (same parameters as in sample wash) each dehydration step. HMDS air-drying agent drop $(250 \mu \mathrm{L})$ on MEB stubs, gold sputter coated for 5 minutes and SEM observation of delicate dinoflagellates.

The preparative technique described for delicate dinoflagellates allows well preservation of important morphological features of the thin thecal surface and plates arrangement of Coolia sp. The present method diminished the distortion and subsequent artifacts observation in SEM micrographs. It does not require osmium tetroxide post-fixation or buffer solution preparation, HMDS drying agent replaces CPD. This protocol is simple, effective and less time consuming [5]. 
References:

[1] JI Goldstein et al., in "Scanning Electron Microscopy and X-Ray Microanalysis. A text for Biologists, Materials Scientisits, and Geologists", $2^{\text {nd }}$ edition. (Plenum Press, New York) p. 576.

[2] L Botes et al., Microscopy Research and Technique 59 (2002), p. 128.

[3] S Won Jung et al., Journal of Applied Phycology 22 (2010), p. 313.

[4] JJ Bozzola et al., in "Electron Microscopy. Principles and Techniques for Biologists", (Jones and Bartlett Publishers, New York) p. 48.

[5] We thank the Marine Biologist Nadia Valeria Herrera Herrera and the students at the Laboratory of Environmental Chemistry for their help and support in sample preparation: Andrea M. García Casillas, and Pamela G. García Santos Reyes.

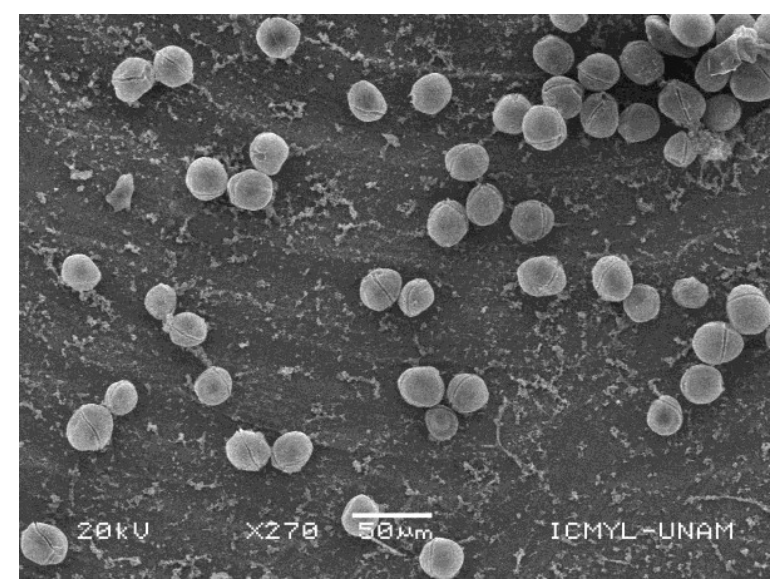

a)

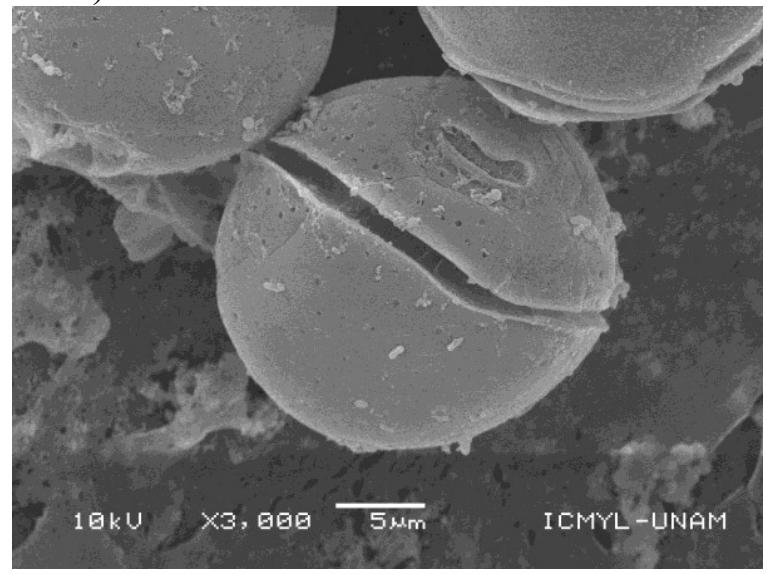

c)

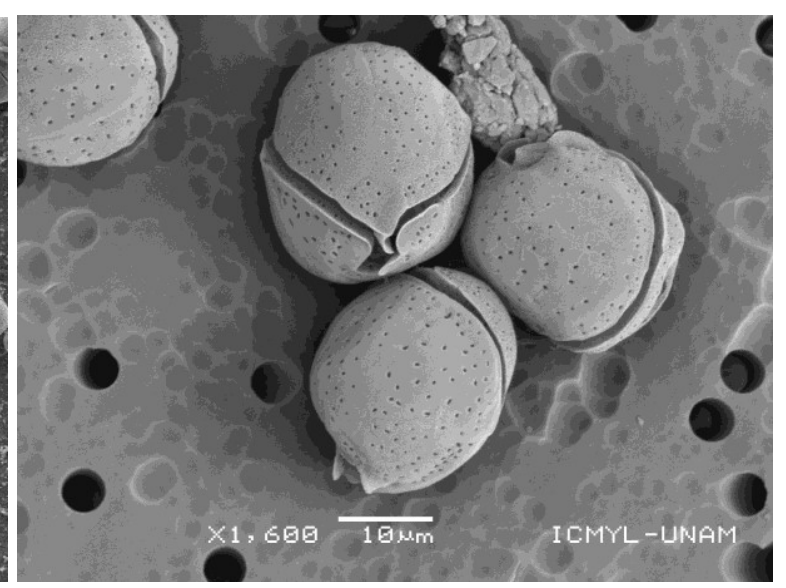

b)

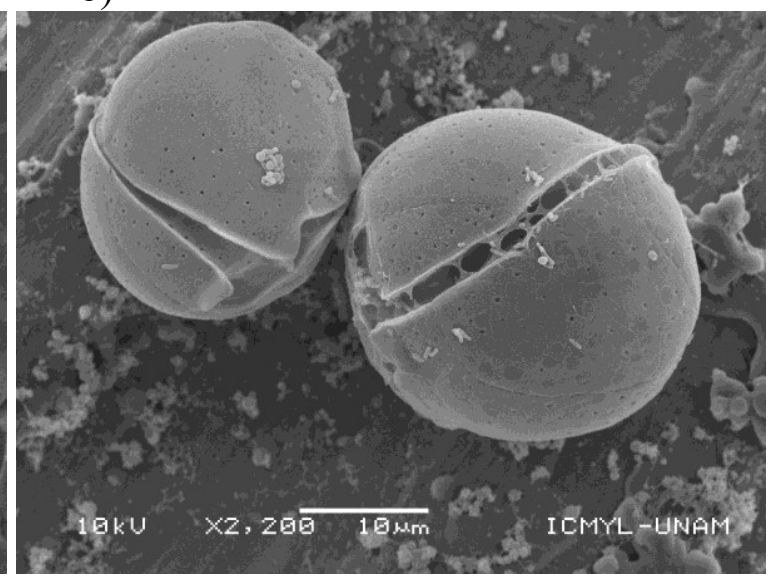

d)

Figure 1. Scanning electron micrographs of Coolia sp. after preparation technique; a) well preservation of dinoflagellates, b) dinoflagellates thin thecal plates c) Po plate, d) dinoflagellates ventral (left) and apical view (rigth). 